\title{
Pralidoxime administered during cardiopulmonary resuscitation facilitates successful resuscitation in a pig model of cardiac arrest
}

Yong Hun Junga, Byung Kook Lee ${ }^{a}$, Kyung Woon Jeung ${ }^{a,}{ }^{*}$, Chun Song Youn ${ }^{b}$, Seong Woo Yun ${ }^{c}$, Tag Heo $^{a}$, Yong II Min ${ }^{a}$

a Department of Emergency Medicine, Chonnam National University Hospital, 42 Jebong-ro, Donggu, Gwangju, Republic of Korea

b Department of Emergency Medicine, Seoul St. Mary's Hospital, The Catholic University of Korea, 222 Banpo-daero, Seocho-gu, Seoul, Republic of Korea

c Department of Emergency Medical Technology, Namseoul university, 91 Daehak-ro, Seonghwan-eup, Sebuk-gu, Cheonan, Chungnam, Republic of Korea

Pralidoxime (2-pyridine aldoxime methyl chloride) is a drug that belongs to a class of chemicals called oximes. Because of its ability to reverse the binding of organophosphate compounds to acetylcholinesterase, pralidoxime has been widely used as an antidote for organophosphate poisoning.

Several studies reported that pralidoxime had a pressor effect besides its antidotal effect against organophosphate poisoning though this is not well recognised. The pressor effect of pralidoxime theoretically should increase CPP, which is a major determinant of myocardial blood flow during CPR, and thus facilitate successful resuscitation

In the present study, we sought to investigate the effects of pralidoxime administered during CPR on CPP, ROSC rate, and short-term survival rate in a pig model of cardiac arrest. We hypothesised that pralidoxime administered during CPR would improve the CPP, ROSC rate, and short-term survival rate compared to a saline placebo.

\section{Methods}

This prospective randomised study was conducted on 22 Yorkshire/Landrace cross pigs weighing $24.9 \pm 2.6 \mathrm{~kg}$. The Animal Care and Use Committee of Chonnam National University approved the protocol for this study (CNU IACUC-H2018-14).

Experimental protocol

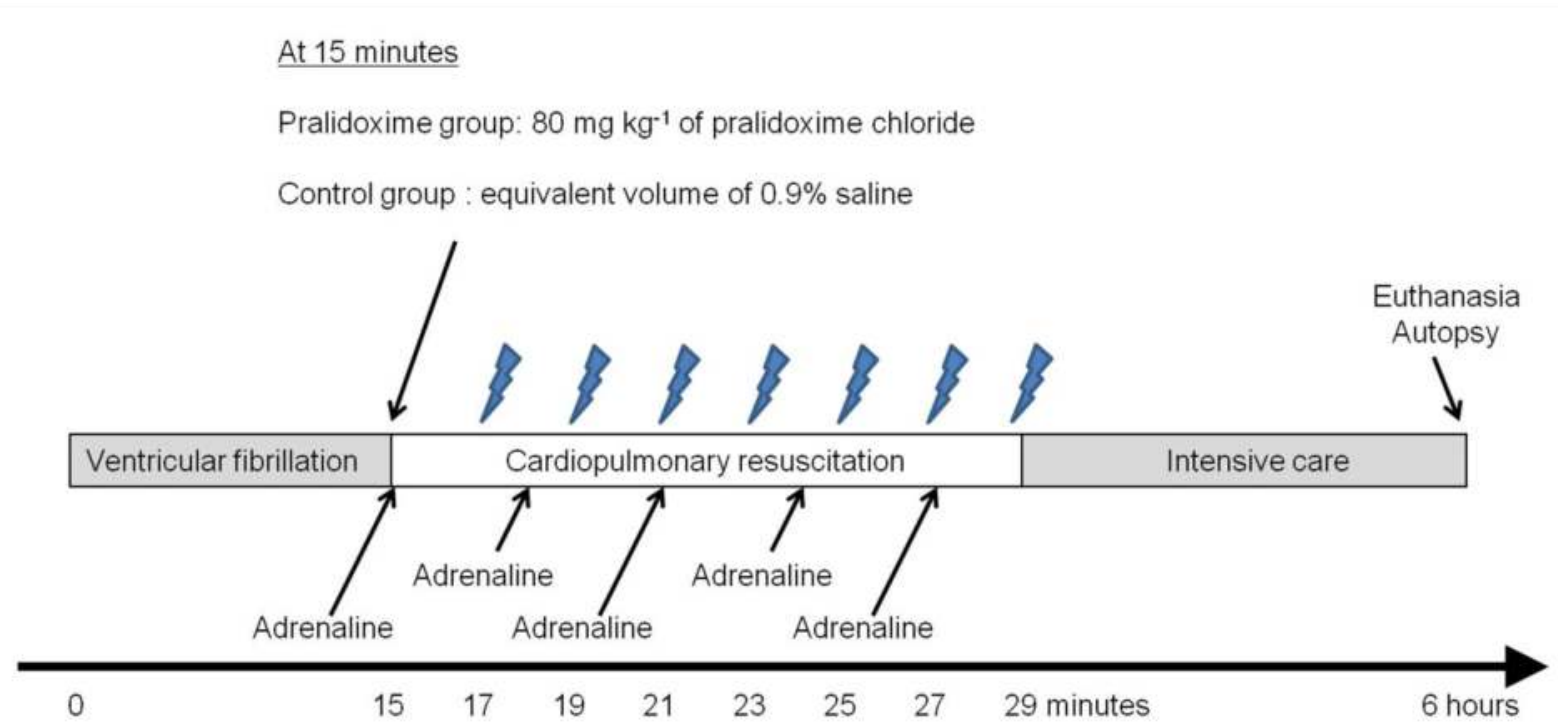

Coinciding with the start of chest compressions, either a saline placebo (control group) or pralidoxime solution (80 $\mathrm{mg} \mathrm{kg}^{-1}$ ) was administered into the RA.
Results

\section{A}

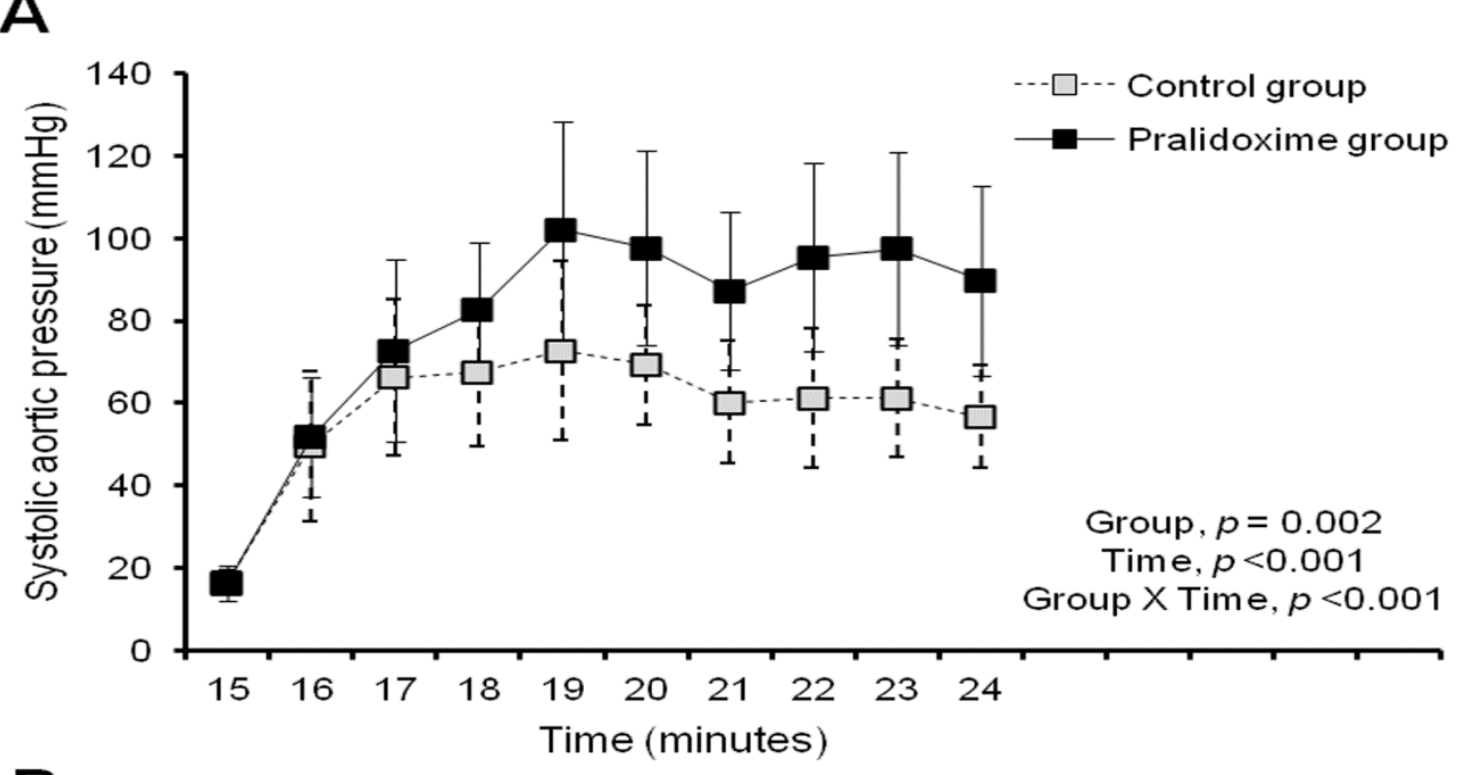

B

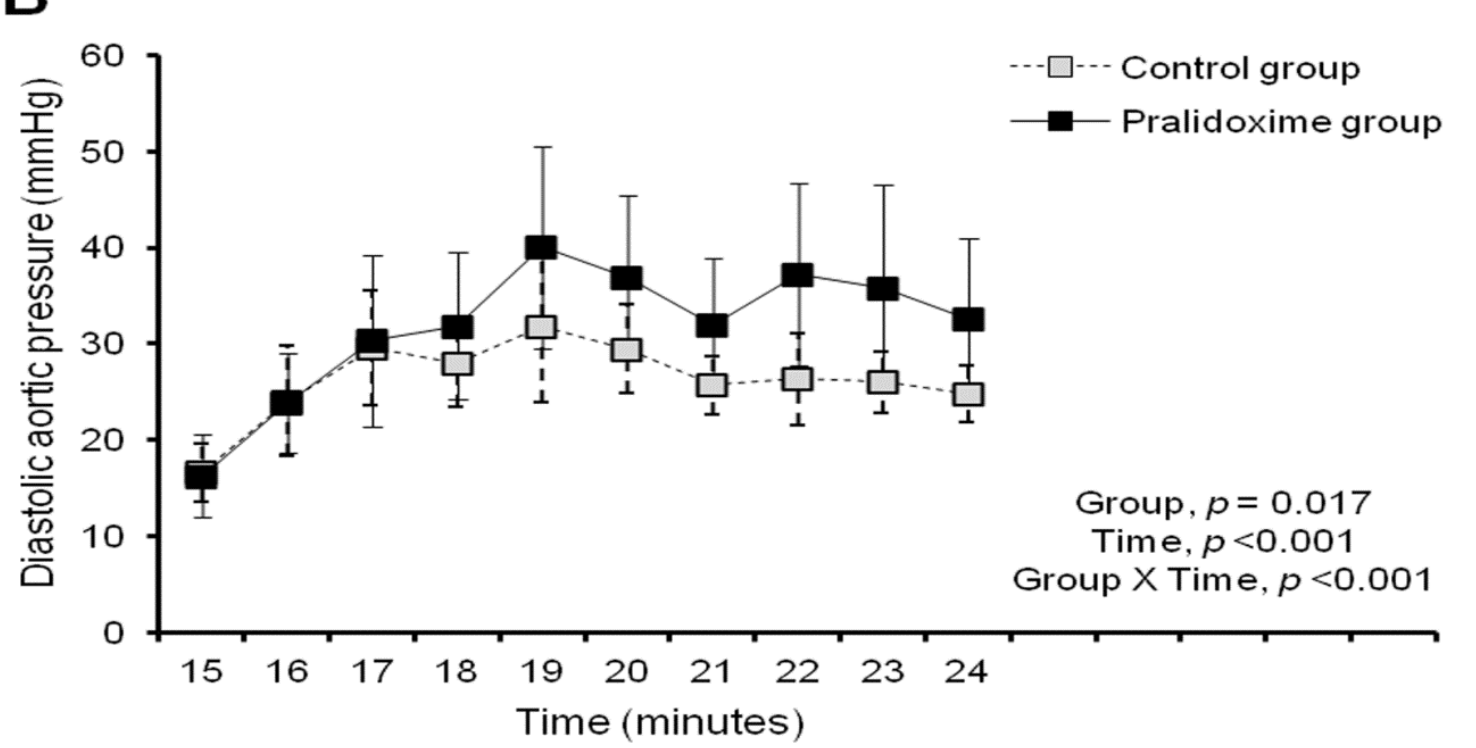

C

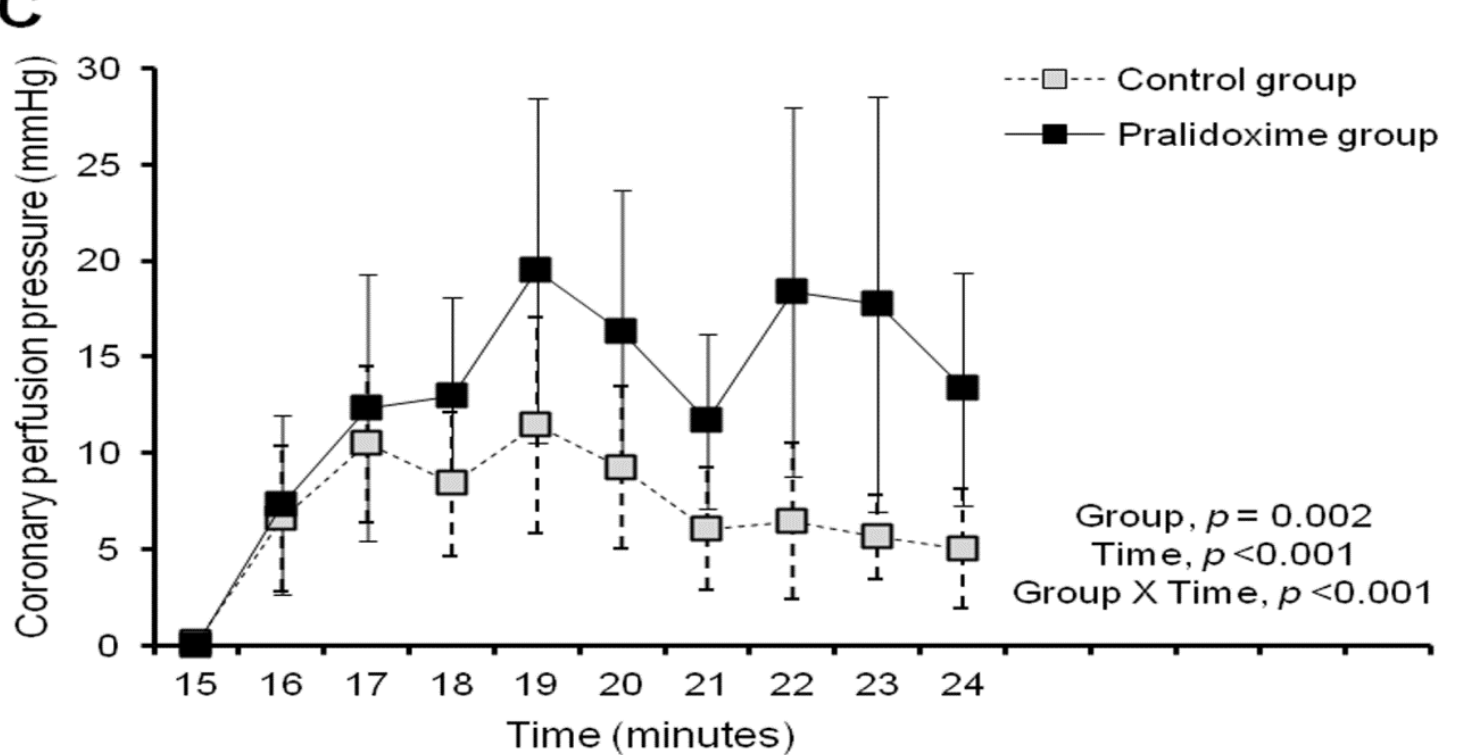

Systolic aortic pressure (A), diastolic aortic pressure (B), and CPP (C) during CPR. Data are presented as means \pm standard deviations. The comparisons of these variables were made using data obtained within 24 minutes after the induction of ventricular fibrillation, as the number of animals still in cardiac arrest markedly decreased after 24 minutes in the pralidoxime group
Nine animals (81.8\%) achieved sustained ROSC in the pralidoxime group, while only three animals $(27.3 \%)$ did in the control group $(p=0.010)$

During the 6-hour intensive care period, one animal in the pralidoxime group died 23 minutes after ROSC due to severe cardiogenic shock refractory to noradrenaline infusion. All the resuscitated animals, except this animal, survived the 6-hour intensive care period (eight animals [72.7\%] in the pralidoxime group versus three animals [27.3\%] in the control group, $p=$ 0.033). During the intensive care period, two of the three control group animals required high-dose noradrenaline $(1.074 \mathrm{mg}$ for 81 minutes and $2.765 \mathrm{mg}$ for 198 minutes, respectively) to maintain mean arterial pressure above $65 \mathrm{mmHg}$, while none of the animals that survived the 6 -hour period in the pralidoxime group required noradrenaline infusion.

\section{Conclusions}

Conclusions Pralidoxime administration during CPR led to significantly higher rates of ROSC and 6-hour survival by improving CPP in a pig model of prolonged cardiac arrest. In view of these results, we believe that pralidoxime has the potential to become an adjuvant drug to potentiate the vasopressor effect of adrenaline and ultimately improve the likelihood of successful resuscitation.

\section{Acknowledgments}

This work was supported by the National Research Foundation of Korea (NRF) grant funded by the Korean government (MSIT) (NRF-2018R1A2B6001388).

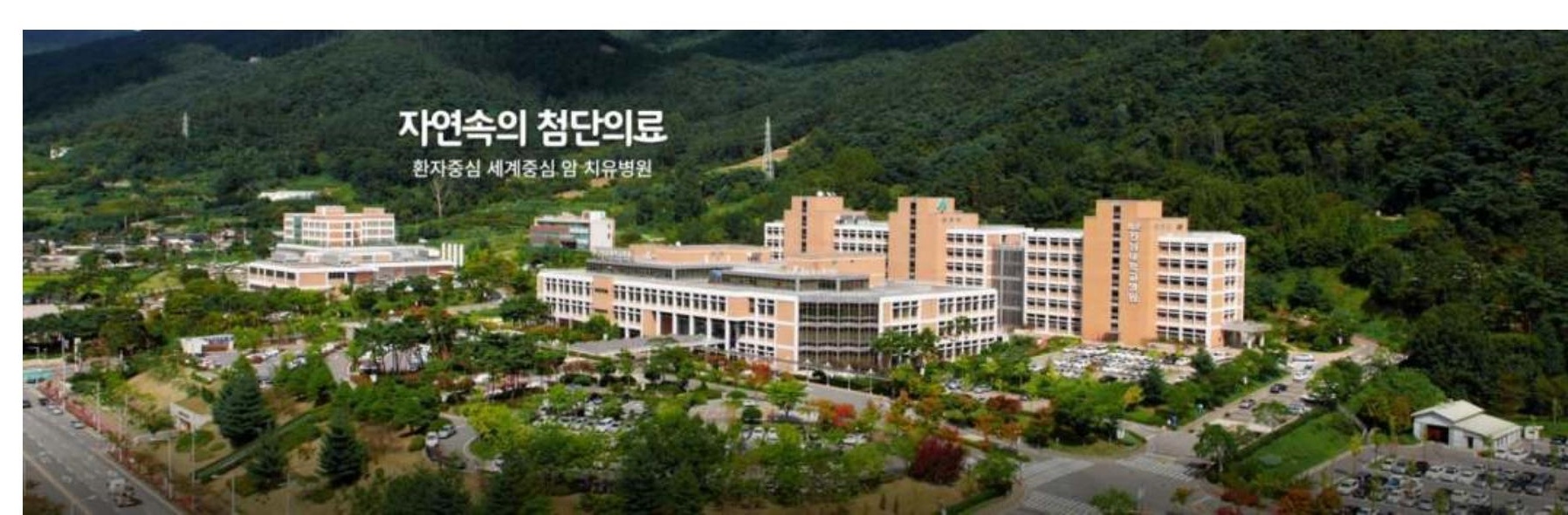

